\title{
Applying the Concept of Peptide Uniqueness to Anti-Polio Vaccination
}

\author{
Darja Kanduc, ${ }^{1}$ Candida Fasano, ${ }^{1}$ Giovanni Capone, ${ }^{1}$ Antonella Pesce Delfino, ${ }^{2}$ \\ Michele Calabrò, ${ }^{2}$ and Lorenzo Polimeno ${ }^{2}$ \\ ${ }^{1}$ Department of Biosciences, Biotechnologies, and Biopharmaceutics, University of Bari, 70126 Bari, Italy \\ ${ }^{2}$ Department of Emergency and Organ Transplantation (DETO), University of Bari, 70124 Bari, Italy
}

Correspondence should be addressed to Darja Kanduc; d.kanduc@biologia.uniba.it

Received 5 February 2015; Accepted 28 April 2015

Academic Editor: Yoshihiko Hoshino

Copyright (C) 2015 Darja Kanduc et al. This is an open access article distributed under the Creative Commons Attribution License, which permits unrestricted use, distribution, and reproduction in any medium, provided the original work is properly cited.

Background. Although rare, adverse events may associate with anti-poliovirus vaccination thus possibly hampering global polio eradication worldwide. Objective. To design peptide-based anti-polio vaccines exempt from potential cross-reactivity risks and possibly able to reduce rare potential adverse events such as the postvaccine paralytic poliomyelitis due to the tendency of the poliovirus genome to mutate. Methods. Proteins from poliovirus type 1, strain Mahoney, were analyzed for amino acid sequence identity to the human proteome at the pentapeptide level, searching for sequences that (1) have zero percent of identity to human proteins, (2) are potentially endowed with an immunologic potential, and (3) are highly conserved among poliovirus strains. Results. Sequence analyses produced a set of consensus epitopic peptides potentially able to generate specific anti-polio immune responses exempt from cross-reactivity with the human host. Conclusion. Peptide sequences unique to poliovirus proteins and conserved among polio strains might help formulate a specific and universal anti-polio vaccine able to react with multiple viral strains and exempt from the burden of possible cross-reactions with human proteins. As an additional advantage, using a peptide-based vaccine instead of current anti-polio DNA vaccines would eliminate the rare post-polio poliomyelitis cases and other disabling symptoms that may appear following vaccination.

\section{Introduction}

Vaccine-associated paralytic poliomyelitis (VAPP) [1] is the consequence of the replication of vaccine-derived polioviruses (VDPVs) that originate by genetic mutations from the strain contained in the oral polio vaccine (OPV). In fact, the poliovirus (PV) genetic mutability [2] appears to be the molecular basis of VAPP, which throws a shadow over the great success represented by vaccination in fighting PV infection $[3,4]$.

Currently, two new monovalent OPVs [5] and the change of the schedule from OPV to the exclusive use of inactivated polio vaccine (IPV) [6] represent options to interrupt PV transmission. However, it has been observed that reduction of exposure to a live attenuated virus such as that contained in OPV will inevitably lead to a decrease in herd immunity to a live microorganism and to natural boosters [7]. Such considerations, along with recent PV infection outbreaks, further complicate the issue of polio eradication [8].

In this scenario, new vaccine formulations and renewed research efforts might help to specifically fight PV infection. Recently, we analyzed the peptide overlap between PV1, strain Mahoney, and the human proteome and described a high extent of peptide sharing involving human proteins linked to fundamental cellular functions [9]. These data appeared to be of interest also because PV has been studied mainly at the nucleotide level [10], and, in general, there is a lack of knowledge of the interaction(s) between $\mathrm{PV}$ and the human host at the peptide/protein level. Actually, phenetic analyses of PV might help define peptide-based therapeutic approaches against PV infection, given that, for example,

(i) one single amino acid (aa) change, that is, His to Tyr at aa position 142 of virion protein 2 (VP2) or Val to 
Ile at aa position 160 of virion protein 1 (VP1), on the capsid surface of PV1 Sabin allows the establishment of persistent infections in HEp-2c cell cultures [11];

(ii) replacement of the Ala residue with Asp at aa position 3 is linked to $50 \%$ loss of virion protein 4 (VP4) precursor myristoylation and severe reduction in specific infectivity [12];

(iii) the 25th aa, Ile, of PV 2C protein interacts with human reticulon 3, a protein involved in viral replication and/or pathogenesis [13].

Moreover, since the 1980s we have known that small aa groupings can play a critical role in neutralizing PV. For example, the discontinuous $\mathrm{E}^{4} \mathrm{~T}^{7} \mathrm{~T} / \mathrm{S}^{8} \mathrm{R}^{9}$ tetrapeptide, which is present in PV VP1, is crucial for neutralizing PV3 by antiPV3 25-1-14 monoclonal antibody (mAb) [14]. Additional examples of short immune determinants in the immune response against PV are the following ones:

(i) A linear heptapeptide (DNNQTSP) is an Ab-binding site mapped to aa residues 164-170 of VP2 [15].

(ii) Short synthetic peptides (e.g., DNTVRET, RSRSES, RSRSESSIESF, and STTNKDK) from PV VP1 prime the immune system of rabbits for a long-lasting, virus-neutralizing IgG $\mathrm{Ab}$ response following a single inoculation of intact virus [16].

(iii) The Immune Epitope Database (IEDB; http://www .immuneepitope.org) [17] includes the continuous linear pentapeptide STTNK (IEDB ID: 61944) $[18,19]$ and the discontinuous pentapeptide $\mathrm{T}^{141} \mathrm{E}^{143} \mathrm{~S}^{312} \mathrm{E}^{315} \mathrm{P}^{417}$ (IEDB ID: 91064) [20] as $\mathrm{PV}$-derived epitopes.

Following the mathematical quantification of pentapeptide sharing between PV1 and human proteins, we reported that 2,040 out of the 2,204 pentamers composing the PV1 proteome are shared with the human proteome for a total of 18,223 matches, including multiple occurrences [9]. In general, a vast peptide sharing with human proteins is a characteristic of viral proteomes [21]. This peptide commonality suggests the existence of common evolutionary links between entities widely different as viruses and Homo sapiens and, in addition, indicates that potential cross-reactivity may affect antivirus vaccine formulations [22] and serological analyses [23]. Hence, it is reasonable to postulate that vaccines based on peptides unique to a virus and absent in the host proteome would guarantee high specificity and, at the same time, eliminate potential cross-reactivity.

Pursuing the objectives of overcoming the difficulties posed by the PV tendency to mutate and eliminating the viral reactivation-related VAPP in order to contribute to the global eradication of poliomyelitis, here we examine PV1 Mahoney polyprotein primary sequence and describe a set of pentapeptides uniquely owned by PV1, endowed with immunologic potential, and conserved among 43 PV strains. We propose that this set of pentapeptides might be used in preclinical and in vivo protocols with the ultimate aim of formulating effective, safe, and universal anti-PV vaccines.

\section{Methods}

The primary aa sequence of human PV1 polyproteins, strain Mahoney (NCBI taxonomic identifier: 12081; Swiss-Prot/ UniProtKB entry: P03300), consisting of 11 viral proteins, 2,209 aa long [25] (further details at http://www.uniprot.org/ uniprot/P03300), was analyzed for aa sequence similarity to the human proteome at the pentapeptide level. In brief, the viral polyprotein was dissected into 5-mers sequentially overlapped by four residues: MGAQV, GAQVS, AQVSS, QVSSQ, VSSQK, and so forth; then, each viral pentapeptide was used as a probe to scan the human proteome for instances of the same pentapeptide, as already described [9]. The similarity analysis used the Protein Information Resource (PIR) peptide match program (http://pir.georgetown.edu/) [26].

PV-derived epitopes were retrieved from the Immune Epitope Database (IEDB; http://www.immuneepitope.org) [17]. Only PV epitopes that had been experimentally validated in the human host were considered in this study.

Consensus peptide sequences were defined by ClustalW multialignment analysis (http://www.uniprot.org/program/ ?query=clustalw\&sort=score) [24] of sequences from $43 \mathrm{PV}$ strains retrieved from UniProt database (http://www.uniprot .org/) on the basis of the following characteristics: (1) described in scientific literature; (2) corresponding to the entire PV polyprotein; (3) derived from PV1 and PV3; (4) derived from PV variants isolated from VAPP or acute flaccid paralysis (AFP) patients or from immunocompromised patients with residual paralysis. Description and references of the $43 \mathrm{PV}$ sequences used for multialignment analysis are reported in detail at http://www.uniprot.org/; the relative Swiss-Prot/UniProtKB entries are P03300, P03301, P03302, P06209, Q9Q281, Q9Q280, Q71AZ9, Q5TLH5, D1YSI9, D1YSJ1, D1YSJ2, D1YSJ3, D1YSJ4, D1YSJ5, D1YSJ6, D1YSJ7, D1YSJ9, D1YSK1, D1YSK2, D1YSK3, D1YSK4, D1YSK5, D1YSK6, D1YSK7, D1YSK8, D1YSK9, D1YSL0, D1YSL1, D1YSL2, D1YSL3, D1YSL4, D1YSL5, D2X673, D8L541, B4YUL3, B4YUL4, Q84792, C5HJY2, C5HJY3, D1GE40, D2E679, D1GE41, and D2XUS9.

\section{Results and Discussion}

3.1. Identification of Pentapeptides Unique to PV Type 1, Strain Mahoney. Using the procedure described under Section 2, we searched the human PV1, strain Mahoney, primary sequence for pentapeptides not shared with human proteins. We used pentapeptides as probes since a pentapeptide is a minimal functional unit in immunology [27], thus representing an appropriate length unit in measuring the qualitative/quantitative parameters of immunological phenomena [28].

Table 1 reports the pentapeptide platform that characterizes PV1 Mahoney polyprotein when compared to the Homo sapiens proteome. We find that 164 pentapeptides are unique to the viral polyprotein and absent in human proteins. In a few instances, viral 5-mers consecutively overlap (Table 1, pentapeptides in bold), thus forming 6-, 7-, and 8-mer stretches (e.g., $\quad \mathrm{PV}_{163-169} \mathrm{QNMYYHY}, \quad \mathrm{PV}_{446-453}$ NYYTHWAG, 
Table 1: Pentapeptides unique to PV type 1, strain Mahoney, and absent in the human proteome.

\begin{tabular}{|c|c|c|c|c|c|c|c|c|c|c|c|}
\hline Pos $^{\mathrm{a}}$ & Sequence $^{\mathrm{b}, \mathrm{c}}$ & $\operatorname{Pos}^{\mathrm{a}}$ & Sequence $^{\mathrm{b}, \mathrm{c}}$ & Pos $^{\mathrm{a}}$ & Sequence ${ }^{\mathrm{b}, \mathrm{c}}$ & Pos $^{\mathrm{a}}$ & Sequence $^{\mathrm{b}, \mathrm{c}}$ & Pos $^{a}$ & Sequence ${ }^{b, c}$ & $\operatorname{Pos}^{\mathrm{a}}$ & Sequence $^{\mathrm{b}, \mathrm{c}}$ \\
\hline 11 & GAHEN & 393 & MIPLN & 746 & PAKWD & 969 & YYPAR & 1399 & CHQPA & 1750 & EIQWM \\
\hline 29 & TINYY & 403 & KNTMD & 748 & KWDDY & 974 & YQSHI & 1403 & ANFKR & 1751 & IQWMR \\
\hline 72 & NIEAC & 404 & NTMDM & 749 & WDDYT & 996 & CHHGV & 1413 & CGKAI & 1752 & QWMRP \\
\hline 73 & IEACG & 408 & MYRVQ & 752 & YTWQT & 1014 & FADIR & 1418 & QLMDK & 1762 & YPIIN \\
\hline 104 & YGRWP & 414 & NDNPH & 763 & FYTYG & 1021 & YAYEE & 1437 & IVNER & 1810 & YVGNK \\
\hline 106 & RWPEY & 436 & LSHTM & 783 & AYSHF & 1084 & ITRNY & 1458 & PIQYK & 1840 & TEQMC \\
\hline 107 & WPEYL & 446 & NYYTH & 784 & YSHFY & 1105 & VSPWQ & 1475 & ECIND & 1849 & MYGTD \\
\hline 130 & CRFYT & 447 & YYTHW & 785 & SHFYD & 1136 & TEACN & 1489 & VRNYC & 1933 & VAMRM \\
\hline 145 & RGWWW & 448 & YTHWA & 835 & KIRVY & 1139 & CNAAK & 1490 & RNYCE & 1935 & MRMAF \\
\hline 146 & GWWWK & 449 & THWAG & 841 & KPKHI & 1188 & STIHQ & 1496 & KGWIV & 1946 & FHKNP \\
\hline 148 & WWKLP & 464 & SMMAT & 847 & VWCPR & 1190 & IHQSC & 1497 & GWIVN & 1947 & HKNPG \\
\hline 149 & WKLPD & 465 & MMATG & 857 & AYYGP & 1204 & FNNVR & 1498 & WIVNI & 1966 & WSKIP \\
\hline 163 & QNMYY & 494 & HVIWD & 863 & VDYKD & 1205 & NNVRW & 1513 & NRAMT & 1979 & AFDYT \\
\hline 164 & NMYYH & 495 & VIWDI & 880 & TYGFG & 1209 & WLSIQ & 1532 & VYVMY & 1983 & TGYDA \\
\hline 165 & MYYHY & 497 & WDIGL & 881 & YGFGH & 1231 & LEHTI & 1534 & VMYKL & 1993 & WFEAL \\
\hline 179 & VQCNA & 507 & MVVPW & 883 & FGHQN & 1291 & FDGYK & 1536 & YKLFA & 2028 & YCVKG \\
\hline 199 & MCLAG & 510 & PWISN & 884 & GHQNK & 1311 & GADMK & 1588 & GEFTM & 2070 & KMIAY \\
\hline 242 & RFCPV & 518 & RQTTN & 894 & GYKIC & 1314 & MKLFC & 1592 & MLGIH & 2073 & AYGDD \\
\hline 244 & CPVDY & 534 & FYQTR & 895 & YKICN & 1326 & FIPPM & 1594 & GIHDN & 2115 & TWENV \\
\hline 271 & TNNCA & 556 & SACND & 897 & ICNYH & 1340 & FTSNY & 1595 & IHDNV & 2131 & KYPFL \\
\hline 292 & KHNNW & 557 & ACNDF & 912 & VSTMW & 1341 & TSNYV & 1596 & HDNVA & 2148 & SIRWT \\
\hline 293 & HNNWG & 558 & CNDFS & 914 & TMWDR & 1368 & RFAFD & 1660 & TETND & 2158 & TQDHV \\
\hline 295 & NWGIA & 568 & DTTHI & 934 & ARCNC & 1369 & FAFDM & 1677 & MFVPV & 2168 & LAWHN \\
\hline 323 & PMCC & 569 & TTHIG & 942 & VYYCE & 1371 & FDMDI & 1699 & RTLMY & 2201 & LYRRW \\
\hline 324 & PMCCE & 588 & MIDNT & 948 & RRKYY & 1388 & DMTMA & 1700 & TLMYN & & \\
\hline 326 & CCEFN & 665 & CVSII & 959 & PTFQY & 1389 & MTMAT & 1701 & LMYNF & & \\
\hline 327 & CEFNG & 708 & RFDME & 961 & FQYME & 1394 & EMCKN & 1702 & MYNFP & & \\
\hline 391 & DTMIP & 735 & QIMYV & 962 & QYMEA & 1398 & NCHQP & 1742 & SYFTQ & & \\
\hline
\end{tabular}

a aa position along the human PV type 1, strain Mahoney, primary sequence.

$\mathrm{b}$ aa sequences given in one-letter code.

${ }^{\mathrm{c}}$ Consecutively overlapping pentapeptides forming 7- and 8-mer stretches unique to PV are given in bold.

$\mathrm{PV}_{783-789}$ AYSHFYD,

$\mathrm{PV}_{1594-1600}$ GIHDNVA, $\quad \mathrm{PV}_{1699-1706}$ RTLMYNFP, and $\mathrm{PV}_{1750-1756}$ EIQWMRP).

3.2. Analysis of the Immunologic Potential of Peptides Unique to $P V$. Next, the pentapeptides described in Table 1 were analyzed for their immunologic potential as follows. PVderived epitopes were retrieved from IEDB, and the epitopes that had been experimentally validated in the human host were analyzed for the presence of pentapeptides unique to PV1 (see Table 1).

As reported in Table 2, the search through IEDB produced a final list of 78 viral epitopes derived from PV1 Mahoney, PV3 Sabin, and PV3 (P3/LEON/37 and P3/LEON $12 \mathrm{~A}$ (1)B). Epitopes derived from PV2 strains were not considered since only data from immunoassays in mice, rats, and/or rabbits were available in IEDB for PV2 strains at the time of this study. Following sequence analysis, it was found that 20 of the 78 epitopes (i.e., IEDB IDs: 31814,48785 , 58511, 59797, 71769, 79272, 79480, 99910, 100138, 100244, 100349, 100382, 100536, 100576, 100631, 100667, 100672, 146181, 146248, and 146390; see IEDB IDs in bold in Table 2) have a total of 12 viral pentapeptides that are absent in the human proteome (pentapeptides in capital letters in Table 2). That is, a first conclusion from Table 2 is that 12 out of the 164 pentapeptides unique to PV1, strain Mahoney, are part of 20 PV epitopic sequences endowed with an immunologic potential in the human host.

Moreover, it can be seen that the 12 unique PV1 pentapeptides are not distributed at random among the PV-derived epitopes. For example, three unique pentapeptides (KWDDY, WDDYT, and YTWQT) overlap each other in the epitope IEDB ID 48785, sequence ppgapvpeKWDDYTWQTssnp (with unique overlapping pentapeptides in capital); the heptapeptide AYSHFYD, formed by three overlapping unique 
TABLE 2: Twelve pentapeptides unique to PV1, Mahoney strain, and absent in the human proteome are distributed among twenty PV-derived epitopes recognized by human sera and/or $\mathrm{T}$ cells.

\begin{tabular}{|c|c|c|c|c|}
\hline IEDB ID $^{\mathrm{a}}$ & Epitope sequence $\mathrm{e}^{\mathrm{b}, \mathrm{c}}$ & PV antigen & PV strain & Immune context \\
\hline 30661 & kevpaltavetgat & VP1 & PV3 Sabin & $\mathrm{B}$ \\
\hline 31814 & klefftysRFDMEltfvvtan & VP1 & PV1 Mahoney & $\mathrm{T}$ \\
\hline 46859 & paltavetgatnpl & VP1 & PV1 Mahoney & B-T \\
\hline 48785 & ppgapvpeKWDDYTWQTssnp & VP1 & PV1 Mahoney & $\mathrm{T}$ \\
\hline 55952 & rsrsessiesf & VP1 & PV1 Mahoney & B \\
\hline 58511 & siFYTYGtaparisvpyvgi & VP1 & PV1 Mahoney & $\mathrm{T}$ \\
\hline 59797 & snAYSHFYDgfskvplkdqs & VP1 & PV1 Mahoney & $\mathrm{T}$ \\
\hline 66978 & tvdnsasttnkdklfavwk & Polyprotein & PV1 Mahoney & $\mathrm{T}$ \\
\hline 71769 & vvndhnptkvtsKIRVYlkp & Polyprotein & PV1 Mahoney & $\mathrm{T}$ \\
\hline 79155 & altlslpkqqdslpdtka & Polyprotein & PV3 (P3/LEON/37 and P3/LEON 12A (1)B) & $\mathrm{T}$ \\
\hline 79160 & atnplapsdtvqtrhvvq & Polyprotein & PV3 (P3/LEON/37 and P3/LEON 12A (1)B) & $\mathrm{T}$ \\
\hline 79186 & dneqpttraqklfam & Polyprotein & PV3 (P3/LEON/37 and P3/LEON 12A (1)B) & $\mathrm{T}$ \\
\hline 79269 & kevpaltavetgatnpla & Polyprotein & PV3 (P3/LEON/37 and P3/LEON 12A (1)B) & $\mathrm{T}$ \\
\hline 79272 & khvrVWCPRppravpyyg & Polyprotein & PV3 (P3/LEON/37 and P3/LEON 12A (1)B) & $\mathrm{T}$ \\
\hline 79318 & nghalnqvyqimyippga & Polyprotein & PV3 (P3/LEON/37 and P3/LEON 12A (1)B) & $\mathrm{T}$ \\
\hline 79350 & qklfamwritykdtv & Polyprotein & PV3 (P3/LEON/37 and P3/LEON 12A (1)B) & $\mathrm{T}$ \\
\hline 79354 & qpttraqklfamwri & Polyprotein & PV3 (P3/LEON/37 and P3/LEON 12A (1)B) & $\mathrm{T}$ \\
\hline 79433 & traqklfamwrityk & Polyprotein & PV3 (P3/LEON/37 and P3/LEON 12A (1)B) & $\mathrm{T}$ \\
\hline 79434 & traqklfamwritykdtv & Polyprotein & PV3 (P3/LEON/37 and P3/LEON 12A (1)B) & $\mathrm{T}$ \\
\hline 79435 & trhvvqrrsrsestiesf & Polyprotein & PV3 (P3/LEON/37 and P3/LEON 12A (1)B) & $\mathrm{T}$ \\
\hline 79436 & tskvriymkpkhvrvw & Polyprotein & PV3 (P3/LEON/37 and P3/LEON 12A (1)B) & $\mathrm{T}$ \\
\hline 79443 & vaiievdneqpttraqkl & Polyprotein & PV3 (P3/LEON/37 and P3/LEON 12A (1)B) & $\mathrm{T}$ \\
\hline 79461 & vrvvndhnptkvtskvri & Polyprotein & PV3 (P3/LEON/37 and P3/LEON 12A (1)B) & $\mathrm{T}$ \\
\hline 79480 & yippgaptpksWDDYTwq & Polyprotein & PV3 (P3/LEON/37 and P3/LEON 12A (1)B) & $\mathrm{T}$ \\
\hline 80446 & argacvtimtvdnpa & VP1 & PV1 Mahoney & $\mathrm{B}$ \\
\hline 81394 & easgpthskeipalt & VP1 & PV1 Mahoney & $\mathrm{B}$ \\
\hline 82831 & gpthskeipaltave & VP1 & PV1 Mahoney & $\mathrm{B}$ \\
\hline 83234 & hskeipaltavetga & VP1 & PV1 Mahoney & $\mathrm{B}$ \\
\hline 88446 & sdtvqtrhvvqhrsr & VP1 & PV1 Mahoney & $\mathrm{B}$ \\
\hline 88495 & sessiesffargacv & VP1 & PV1 Mahoney & B \\
\hline 99863 & aaparisvpyvgla & Polyprotein & PV3 Sabin & B \\
\hline 99886 & ahskevpaltavet & Polyprotein & PV3 Sabin & B \\
\hline 99901 & arisvpyvglanay & Polyprotein & PV3 Sabin & B \\
\hline 99910 & AYSHFYDgfakvpl & Polyprotein & PV3 Sabin & B \\
\hline 99933 & dfgvlavrvvndhn & Polyprotein & PV3 Sabin & B \\
\hline 99963 & dtvqlrrkleffty & Polyprotein & PV3 Sabin & B \\
\hline 100029 & famwritykdtvql & Polyprotein & PV3 Sabin & B \\
\hline 100101 & hfydgfakvplktd & Polyprotein & PV3 Sabin & B \\
\hline 100117 & hnptkvtskvriym & Polyprotein & PV3 Sabin & B \\
\hline 100138 & iFYTYGaaparisv & Polyprotein & PV3 Sabin & B \\
\hline 100244 & lanAYSHFYDgfak & Polyprotein & PV3 Sabin & B \\
\hline 100349 & npsiFYTYGaapar & Polyprotein & PV3 Sabin & B \\
\hline 100382 & pkhvrVWCPRppra & Polyprotein & PV3 Sabin & B \\
\hline 100391 & psdtvqtrhvvqrr & Polyprotein & PV3 Sabin & B \\
\hline 100425 & qklfamwritykdt & Polyprotein & PV3 Sabin & B \\
\hline 100430 & qlrrklefftysrf & Polyprotein & PV3 Sabin & B \\
\hline 100439 & qpttraqklfamwr & Polyprotein & PV3 Sabin & B \\
\hline 100482 & rppravpyygpgvd & Polyprotein & PV3 Sabin & $\mathrm{B}$ \\
\hline 100492 & samtvddfgvlavr & Polyprotein & PV3 Sabin & $\mathrm{B}$ \\
\hline
\end{tabular}


TABLe 2: Continued.

\begin{tabular}{|c|c|c|c|c|}
\hline IEDB ID $^{\mathrm{a}}$ & Epitope sequence $^{\mathrm{b}, \mathrm{c}}$ & PV antigen & PV strain & Immune context \\
\hline 100504 & sevaqgaltlslpk & Polyprotein & PV3 Sabin & $\mathrm{B}$ \\
\hline 100536 & svpyvglanAYSHF & Polyprotein & PV3 Sabin & B \\
\hline 100559 & tkvtskvriymkpk & Polyprotein & PV3 Sabin & B \\
\hline 100573 & traqklfamwrity & Polyprotein & PV3 Sabin & B \\
\hline 100575 & tskvriymkpkhvr & Polyprotein & PV3 Sabin & B \\
\hline 100576 & tssnpsiFYTYGaa & Polyprotein & PV3 Sabin & $\mathrm{B}$ \\
\hline 100580 & tvddfgvlavrvvn & Polyprotein & PV3 Sabin & B \\
\hline 100583 & tvqtrhvvqrrsrs & Polyprotein & PV3 Sabin & B \\
\hline 100585 & twqtssnpsifyty & Polyprotein & PV3 Sabin & $\mathrm{B}$ \\
\hline 100586 & tygaaparisvpyv & Polyprotein & PV3 Sabin & B \\
\hline 100587 & tykdtvqlrrklef & Polyprotein & PV3 Sabin & B \\
\hline 100613 & vlavrvvndhnptk & Polyprotein & PV3 Sabin & B \\
\hline 100619 & vndhnptkvtskvr & Polyprotein & PV3 Sabin & B \\
\hline 100628 & vriymkpkhvrvwc & Polyprotein & PV3 Sabin & $\mathrm{B}$ \\
\hline 100630 & vrvvndhnptkvts & Polyprotein & PV3 Sabin & B \\
\hline 100631 & vrVWCPRppravpy & Polyprotein & PV3 Sabin & $\mathrm{B}$ \\
\hline 100638 & wcprppravpyygp & Polyprotein & PV3 Sabin & B \\
\hline 100644 & writykdtvqlrrk & Polyprotein & PV3 Sabin & B \\
\hline 100667 & ymkpkhvrVWCPRp & Polyprotein & PV3 Sabin & B \\
\hline 100672 & yvglanAYSHFYDg & Polyprotein & PV3 Sabin & $\mathrm{B}$ \\
\hline 146178 & ayappgaqpptsrk & Polyprotein & PV3 Sabin & B \\
\hline 146181 & cgSMMATGkilvay & Polyprotein & PV3 Sabin & B \\
\hline 146248 & flfcgSMMATGkil & Polyprotein & PV3 Sabin & $\mathrm{B}$ \\
\hline 146311 & hqgalgvfaipeyc & Polyprotein & PV3 Sabin & B \\
\hline 146333 & ilvayappgaqppt & Polyprotein & PV3 Sabin & B \\
\hline 146390 & kftflfcgSMMATG & Polyprotein & PV3 Sabin & B \\
\hline 146494 & phqiinlrtnnsat & Polyprotein & PV3 Sabin & B \\
\hline 146496 & ppgaqpptsrkeam & Polyprotein & PV3 Sabin & $\mathrm{B}$ \\
\hline 146516 & ravpyygpgvdyrn & Polyprotein & PV3 Sabin & B \\
\hline
\end{tabular}

${ }^{\mathrm{a}} \mathrm{PV}$-derived epitopes are listed according to increasing IEDB ID number. Further details and reference(s) on each IEDB ID are reported at http://www.immuneepitope.org [17].

${ }^{\mathrm{b}}$ Only PV-derived epitopes that had been experimentally validated in the human host are reported.

${ }^{c}$ The twenty PV-derived epitopes (and related IDs) containing PV-pentapeptide(s) absent in the human proteome are in bold, with the pentapeptide(s) absent in the human proteome given in capital.

pentapeptides shifted by one residue, characterizes four PV-derived epitopes (IEDB IDs: 59797, 99910, 100244, and 100672); the hexapeptide SMMATG formed by two overlapped 5-mers is present in three epitopes (IEDB IDs: 146181, 146248, and 146390).

Theoretically, vaccines based on such PV epitopic peptides (i.e., KWDDYTWQT, AYSHFYD, and SMMATG) might evoke highly specific anti-PV immune responses exempt of possible collateral cross-reactions in the human host.

3.3. Identification of Consensus Pentapeptides Unique to PVs and Endowed with Immunologic Potential. We reasoned that using epitopic peptides unique to the virus and conserved among PV strains might help develop a global anti-PV peptide-based vaccination protocol. Such an approach would be of special importance in providing an effective and wide coverage to the human population worldwide, thus allowing reaching the goal of PV eradication $[29,30]$. To this aim, we searched for unique conserved sequences by analysing a set of $43 \mathrm{PV}$ polyproteins selected as described under Section 2 and comprehending also PV variants isolated from faeces of VAPP or AFP or from immunocompromised patient(s) with residual paralysis. The $43 \mathrm{PV}$ polyprotein sequences were aligned using multialignment ClustalW program [24] and the peptide sequences present in PV-derived epitopes (see Table 2) and absent in the human proteome were localized.

Table 3 shows that seven potentially immunogenic peptides (in the order $\mathrm{PV}_{465-469}$ MMATG, $\mathrm{PV}_{708-712}$ RFDME, PV $_{752-756}$ DYTWQT, PV ${ }_{763-767}$ FYTYG, PV ${ }_{783-789}$ AYSHFYD, $\mathrm{PV}_{835-839} \mathrm{KIRVY}$, and $\mathrm{PV}_{847-851}$ VWCPR), derived from epitopes corresponding to (or formed of) pentapeptides unique 
TABLE 3: Conservativeness of epitopic PV peptide regions among 43 PV strains and variants.

\begin{tabular}{|c|c|c|c|c|c|c|c|c|}
\hline PV strain & $\begin{array}{l}\text { UniProt/Swiss-Prot } \\
\text { entry }\end{array}$ & aa $\operatorname{Pos} 464$ & aa Pos 708 & aa $\operatorname{Pos} 748$ & $\begin{array}{l}\text { aa Pos } \\
763\end{array}$ & $\begin{array}{c}\text { aa Pos } \\
783\end{array}$ & $\begin{array}{l}\text { aa Pos } \\
835\end{array}$ & $\begin{array}{c}\text { aa Pos } \\
847\end{array}$ \\
\hline PV1 Mahoney & $\mathrm{P} 03300$ & FMMATG & RFDME & KWDDYTWQT & FYTYG & AYSHFYD & KIRVY & VWCPR \\
\hline PV1 Sabin & P03301 & SMMATG & RFDME & KWDDYTWQT & FYTYG & AYSHFYD & KIRVY & VWCPR \\
\hline PV3 P3/Leon/37 & P03302 & SMMATG & RFDME & sWDDYTWQT & FYTYG & AYSHFYD & KvRiY & VWCPR \\
\hline PV3 23127 & P06209 & SMMATG & RFDME & sWDDYTWQT & FYTYG & AYSHFYD & KvRvY & VWCPR \\
\hline PV1 isolated & Q9Q281 & SMMATG & RFDME & KWDDYTWQT & FYTYG & AYSHFYD & KvRVY & VWCPR \\
\hline PV1 isolated & Q9Q280 & SMMATG & RFDME & KWDDYTWQT & FYTYG & AYSHFYD & KIRVY & VWCPR \\
\hline PV1 isolated & Q71AZ9 & SMMATG & RFDME & KWDDYTWQT & FYTYG & AYSHFYD & KIRVY & VWCPR \\
\hline PV1 isolated & Q5TLH5 & SMMATG & RFDME & KWDDYTWQT & FYTYG & AYSHFYD & KIRVY & VWCPR \\
\hline PV1-HAI01008C2 & D1YSI9 & SMMATG & RFDME & KWDDYTWQT & FYTYG & AYSHFYD & KIRVY & VWCPR \\
\hline PV1-HAI01009 & D1YSJ1 & SMMATG & RFDME & KWDDYTWQT & FYTYG & AYSHFYD & KIRVY & VWCPR \\
\hline PV1-HAI01008 & D1YSJ2 & SMMATG & & KWDDYTWQT & FYTYG & AYSHFYD & KIRVY & VWCPR \\
\hline PV1-HAI01002 & D1YSJ3 & SMMATG & RFDME & KWDDYTWQT & FYTYG & AYSHFYD & KIRVY & VWCPR \\
\hline PV1-HAI01001 & D1YSJ4 & SMMATG & RFDME & KWDDYTWQT & FYTYG & AYSHFYD & KIRVY & VWCPR \\
\hline PV1-DOR01012 & D1YSJ5 & SMMATG & RFDME & KWDDYTWQT & FYTYG & AYSHFYD & KIRVY & VWCPR \\
\hline PV1-DOR01002C & D1YSJ6 & SMMATG & RFDME & KWDDYTWQT & FYTYG & AYSHFYD & KIRVY & VWCPR \\
\hline PV1-DOR01002 & D1YSJ7 & SMMATG & RFDME & KWDDYTWQT & FYTYG & AYSHFYD & KIRVY & VWCPR \\
\hline PV1-DOR01001C1 & D1YSJ9 & SMMATG & RFDME & KWDDYTWQT & FYTYG & AYSHFYD & KIRVY & VWCPR \\
\hline PV1-DOR00042C2 & D1YSK1 & & & KWDDYTWQT & FYTYG & AYSHFYD & KIRVY & VWCPR \\
\hline PV1-DOR00042C1 & D1YSK2 & & & KWDDYTWQT & FYTYG & AYSHFYD & & VWCPR \\
\hline PV1-DOR00042 & & & & KWDDYTWQT & FYTYG & AYSHFYD & & VWCPR \\
\hline PV1-DOR00041C3 & D1YSK4 & SMMATG & & KWDDYTWQT & FYTYG & AYSHFYD & KIRVY & VWCPR \\
\hline PV1-DOR00041C2 & D1YSK5 & SMMATG & & KWDDYTWQT & FYTYG & AYSHFYD & KIRVY & VWCPR \\
\hline PV1-DOR00044 & D1YSK6 & SMMATG & & KWDDYTWQT & FYTYG & AYSHFYD & KIRVY & VWCPR \\
\hline PV1-DOR00028C & D1YSK7 & SMMATG & RFDME & KWDDYTWQT & FYTYG & AYSHFYD & KIRVY & VWCPR \\
\hline PV1-DOR00028 & D1YSK8 & SMMATG & RFDME & KWDDYTWQT & FYTYG & AYSHFYD & KIRVY & VWCPR \\
\hline PV1-DOR00023C & D1YSK9 & SMMATG & RFDME & KWDDYTWQT & FYTYG & AYSHFYD & KIRVY & VWCPR \\
\hline PV1-DOR00025 & D1YSL0 & SMMATG & RFDME & KWDDYTWQT & FYTYG & AYSHFYD & KIRVY & VWCPR \\
\hline PV1-DOOR24 & D1YSL1 & SMMATG & & KWDDYTWQT & FYTYG & AYSHFYD & KIRVY & VWCPR \\
\hline PV1-DOR00015 & D1YSL2 & SMMATG & RFDME & KWDDYTWQT & FYTYG & AYSHFYD & KIRVY & VWCPR \\
\hline PV1-DOR00016 & D1YSL3 & SMMATG & & KWDDYTWQT & FYTYG & AYSHFYD & KIRVY & VWCPR \\
\hline PV1-HAI01013all & D1YSL4 & SMMATG & & KWDDYTWQT & FYTYG & AYSHFYD & KIRVY & VWCPR \\
\hline PV1-HAI01015 & D1YSL5 & SMMATG & RFDME & KWDDYTWQT & FYTYG & AYSHFYD & KIRVY & VWCPR \\
\hline PV1-S302 & D2X673 & SMMATG & RFDME & KWDDYTWQT & FYTYG & AYSHFYD & KIRVY & VWCPR \\
\hline CHN8184/GZ/CHN/2004 & D8L541 & SMMATG & RFDME & KWDDYTWQT & FYTYG & AYSHFYD & KIRVY & VWCPR \\
\hline PV3 isolated & B4YUL3 & SMMATG & RFDME & sWDDYTWQT & FYTYG & AYSHFYD & KvRVY & VWCPR \\
\hline PV3 isolated & B4YUL4 & SMMATG & RFDME & sWDDYTWQT & FYTYG & AYSHFYD & KvRVY & VWCPR \\
\hline $\begin{array}{l}\text { PV3 (vacc.StrainSabin3 } \\
(\text { Leon 12alb)) }\end{array}$ & Q84792 & SMMATG & RFDME & sWDDYTWQT & FYTYG & AYSHFYD & KvRiY & VWCPR \\
\hline PV3-33239 & C5HJY2 & SMMATG & RFDME & sWDDYTWQT & FYTYG & AYSHFYD & KvRiY & VWCPR \\
\hline PV3-31974 & С5HJY3 & SMMATG & RFDME & sWDDYTWQT & FYTYG & AYSHFYD & KvRiY & VWCPR \\
\hline PV3-FIN84-60212 & D1GE40 & SMMATG & RFDME & sWDDYTWQT & FYTYG & AYSHFYD & KvRiY & VWCPR \\
\hline PV3-SWI10947 & D2E679 & SMMATG & RFDME & sWDDYTWQT & FYTYG & AYSHFYD & KvRiY & VWCPR \\
\hline PV3-FIN84-2493 & D1GE41 & SMMATG & RFDME & sWDDYTWQT & FYTYG & AYSHFYD & KvRiY & VWCPR \\
\hline PV3-P3/Jinan/1/09 & D2XUS9 & SMMATG & RFDME & sWDDYTWQT & FYTYG & AYSHFYD & KvRiY & VWCPR \\
\hline
\end{tabular}

The 43 PV sequences were aligned using ClustalW multialignment program (http://www.uniprot.org/align/) [24]. The analyzed PV sequences and related references are described at http://www.uniprot.org/ and reported by Swiss-Prot/UniProtKB accession number. Peptide sequences present in PV-derived epitopes (see Table 2) and absent in the human proteome were localized along the 43 aligned PV sequences and analyzed for conservativeness. Peptide sequences are indicated by their position along the PV polyprotein. Mutated aa residues are in lower case. 
to PV1 (see Tables 1 and 2), have $100 \%$ conservation among the $43 \mathrm{PV}$ strains/variants under analysis. The seven potentially immunogenic unique peptide sequences had the same level of conservativeness in PV2 derived strains (data not shown).

\section{Conclusion}

Anti-PV immunization has been one of the major public health measures of the last century. International campaigns to eliminate polio reduced the incidence of this disease in the world. However, problematic issues remain, such as the tendency of the PV genome to mutate, the potential risk to develop postvaccine paralytic poliomyelitis, and the difficulty to completely eradicate PV infection in the world. In fact, according to the World Health Organization, in 2012, still three countries in the world remain polio-endemic: Nigeria, Pakistan, and Afghanistan [31].

The present data propose the concept of sequence uniqueness as a tool to define specific immunotherapies exempt of collateral effects [22] and describe a methodology to identify PV peptides that have zero percent identity to human proteins, are endowed with an immunologic potential, and are highly conserved among PV strains (e.g., $\mathrm{PV}_{465-469}$ MMATG, PV ${ }_{708-712}$ RFDME, PV ${ }_{752-756}$ DYTWQT, PV $_{763-767}$ FYTYG, PV ${ }_{783-789}$ AYSHFYD, PV ${ }_{835-839}$ KIRVY, and $\mathrm{PV}_{847-851}$ VWCPR; see Table 3). Importantly, polio peptide sequences alternate through the human proteome with a frequency versus rarity pattern that characterizes other pathogens too [32-35].

Theoretically, such viral consensus epitopic peptides appear to be ideal tools to generate anti-PV immune responses promising of high specificity, thus avoiding serological cross-reactivity between human polyomaviruses [23], as well as possible cross-reactions with the human host [22]. As an example, a construct composed of the coding frames corresponding to the immunogenic consensus sequences described above (Table 2) might determine a specific anti-PV immune response and, at the same time, by being based on peptides, might eliminate the issues inherent to the tendency of the PV genome to mutate (i.e., VAPP). Such viral peptide sequences might also be used in passive anti-PV immunotherapies, that is, to produce specific antibodies capable of reacting with intact viral protein antigens.

Actually, the present report is intended to represent a first approach to preclinical and animal studies. As a matter of fact, the solidity of a large body of theoretical and in silico data is the mandatory basis to design in vivo experimentation and validation protocols especially when considering that (i) although monkeys can be experimentally infected, humans are the only known natural hosts of poliovirus; (ii) small animal models for testing polio pathogenesis mainly relate to transgenic mice to express a human receptor to poliovirus [36]; and, moreover, (iii) current laws on in vivo experimentation are increasingly restrictive.

\section{Conflict of Interests}

The authors declare that this paper is based on research that was not funded entirely or partially by an outside source.

\section{Authors' Contribution}

Darja Kanduc proposed the original idea, supervised the work, interpreted the data, and wrote the paper. All authors contributed to the computational analyses; Giovanni Capone also contributed to the project definition. All authors discussed and approved the paper.

\section{References}

[1] T. J. John, "A developing country perspective on vaccineassociated paralytic poliomyelitis," Bulletin of the World Health Organization, vol. 82, no. 1, pp. 53-57, 2004.

[2] C. Runckel, O. Westesson, R. Andino, and J. L. DeRisi, "Identification and manipulation of the molecular determinants influencing poliovirus recombination," PLoS Pathogens, vol. 9, no. 2, Article ID e1003164, 2013.

[3] R. J. D. Tebbens, M. A. Pallansch, J.-H. Kim et al., "Oral poliovirus vaccine evolution and insights relevant to modeling the risks of circulating vaccine-derived polioviruses (cVDPVs)," Risk Analysis, vol. 33, no. 4, pp. 680-702, 2013.

[4] W. R. Dowdle, E. De Gourville, O. M. Kew, M. A. Pallansch, and D. J. Wood, "Polio eradication: the OPV paradox," Reviews in Medical Virology, vol. 13, no. 5, pp. 277-291, 2003.

[5] R. B. Aylward and C. Maher, "Interrupting poliovirus transmission-new solutions to an old problem," Biologicals, vol. 34, no. 2, pp. 133-139, 2006.

[6] S. C. Arya and N. Agarwal, "Re: world wide experience with inactivated poliovirus vaccine," Vaccine, vol. 27, no. 1, p. 1, 2009.

[7] M.-C. Bonnet and A. Dutta, "World wide experience with inactivated poliovirus vaccine," Vaccine, vol. 26, no. 39, pp. 4978-4983, 2008.

[8] B. T. Mayer, J. N. S. Eisenberg, C. J. Henry, M. G. M. Gomes, E. L. Ionides, and J. S. Koopman, "Successes and shortcomings of polio eradication: a transmission modeling analysis," American Journal of Epidemiology, vol. 177, no. 11, pp. 1236-1245, 2013.

[9] G. Novello, G. Capone, C. Fasano, S. L. Bavaro, A. N. Polito, and D. Kanduc, "A quantitative description of the peptide sharing between poliovirus and Homo sapiens," Immunopharmacology and Immunotoxicology, vol. 34, no. 3, pp. 373-378, 2012.

[10] E. Cherkasova, M. Laassri, V. Chizhikov et al., "Microarray analysis of evolution of RNA viruses: evidence of circulation of virulent highly divergent vaccine-derived polioviruses," Proceedings of the National Academy of Sciences of the United States of America, vol. 100, no. 16, pp. 9398-9403, 2003.

[11] I. Pelletier, G. Duncan, and F. Colbère-Garapin, "One amino acid change on the capsid surface of poliovirus sabin 1 allows the establishment of persistent infections in HEp-2c cell cultures," Virology, vol. 241, no. 1, pp. 1-13, 1998.

[12] N. Moscufo, J. Simons, and M. Chow, "Myristoylation is important at multiple stages in poliovirus assembly," Journal of Virology, vol. 65, no. 5, pp. 2372-2380, 1991.

[13] W.-F. Tang, S.-Y. Yang, B.-W. Wu et al., "Reticulon 3 binds the $2 \mathrm{C}$ protein of enterovirus 71 and is required for viral replication," The Journal of Biological Chemistry, vol. 282, no. 8, pp. 5888$5898,2007$. 
[14] D. M. A. Evans, P. D. Minor, G. S. Schild, and J. W. Almond, "Critical role of an eight-amino acid sequence of VP1 in neutralization of poliovirus type 3," Nature, vol. 304, no. 5925, pp. 459-462, 1983.

[15] K. J. Wiegers, K. Wetz, and R. Dernick, "Molecular basis for linkage of a continuous and discontinuous neutralization epitope on the structural polypeptide VP2 of poliovirus type 1," Journal of Virology, vol. 64, no. 3, pp. 1283-1289, 1990.

[16] E. A. Emini, B. A. Jameson, and E. Wimmer, "Priming for and induction of anti-poliovirus neutralizing antibodies by synthetic peptides," Nature, vol. 304, no. 5928, pp. 699-703, 1983.

[17] R. Vita, L. Zarebski, J. A. Greenbaum et al., "The immune epitope database 2.0," Nucleic Acids Research, vol. 38, supplement 1, pp. D854-D862, 2010.

[18] K. Wiegers, H. Uhlig, and R. Dernick, "N-AgIB of poliovirus type 1: a discontinuous epitope formed by two loops of VP1 comprising residues 96-104 and 141-152," Virology, vol. 170, no. 2, pp. 583-586, 1989.

[19] R. Ketterlinus and K. Wiegers, "Mapping of antigenic domains in poliovirus VP1 involved in structural rearrangements during virus morphogenesis and antigenic alterations of the virion," Virology, vol. 204, no. 1, pp. 27-37, 1994.

[20] C. Reynolds, G. Page, H. Zhou, and M. Chow, "Identification of residues in VP2 that contribute to poliovirus neutralization antigenic site 3B," Virology, vol. 184, no. 1, pp. 391-396, 1991.

[21] D. Kanduc, A. Stufano, G. Lucchese, and A. Kusalik, "Massive peptide sharing between viral and human proteomes," Peptides, vol. 29, no. 10, pp. 1755-1766, 2008.

[22] D. Kanduc, "Peptide cross-reactivity: the original sin of vaccines," Frontiers in Bioscience, vol. 4, no. 4, pp. 1393-1401, 2012.

[23] U. Moens, M. van Ghelue, X. Song, and B. Ehlers, "Serological cross-reactivity between human polyomaviruses," Reviews in Medical Virology, vol. 23, no. 4, pp. 250-264, 2013.

[24] J. D. Thompson, D. G. Higgins, and T. J. Gibson, "CLUSTAL $\mathrm{W}$ : improving the sensitivity of progressive multiple sequence alignment through sequence weighting, position-specific gap penalties and weight matrix choice," Nucleic Acids Research, vol. 22, no. 22, pp. 4673-4680, 1994.

[25] V. R. Racaniello and D. Baltimore, "Molecular cloning of poliovirus CDNA and determination of the complete nucleotide sequence of the viral genome," Proceedings of the National Academy of Sciences of the United States of America, vol. 78, no. 8, pp. 4887-4891, 1981.

[26] C. H. Wu, L.-S. Yeh, H. Huang et al., "The protein information resource," Nucleic Acids Research, vol. 31, no. 1, pp. 345-347, 2003.

[27] D. Kanduc, "Homology, similarity, and identity in peptide epitope immunodefinition," Journal of Peptide Science, vol. 18, no. 8, pp. 487-494, 2012.

[28] D. Kanduc, "Pentapeptides as minimal functional units in cell biology and immunology," Current Protein \& Peptide Science, vol. 14, no. 2, pp. 111-120, 2013.

[29] N. C. Grassly, "The final stages of the global eradication of poliomyelitis," Philosophical Transactions of the Royal Society of London, Series B: Biological Sciences, vol. 368, no. 1623, Article ID 20120140, 2013.

[30] P. Minor, "The polio endgame," Human Vaccines \& Immunotherapeutics, vol. 10, no. 7, pp. 2106-2108, 2014.

[31] Z. A. Bhutta, "Conflict and polio: winning the polio wars," Journal of the American Medical Association, vol. 310, no. 9, pp. 905-906, 2013.
[32] D. Kanduc, L. Tessitore, G. Lucchese, A. Kusalik, E. Farber, and F. M. Marincola, "Sequence uniqueness and sequence variability as modulating factors of human anti-HCV humoral immune response," Cancer Immunology, Immunotherapy, vol. 57, no. 8, pp. 1215-1223, 2008.

[33] G. Lucchese, A. Stufano, and D. Kanduc, "Proposing lowsimilarity peptide vaccines against Mycobacterium tuberculosis," Journal of Biomedicine and Biotechnology, vol. 2010, Article ID 832341, 8 pages, 2010.

[34] G. Capone, G. Lucchese, M. Calabrò, and D. Kanduc, "West Nile virus diagnosis and vaccination: using unique viral peptide sequences to evoke specific immune responses," Immunopharmacology and Immunotoxicology, vol. 35, no. 1, pp. 64-70, 2013.

[35] A. Lucchese, A. Guida, G. Capone, M. Petruzzi, D. Lauritano, and R. Serpico, "Designing a peptide-based vaccine against Porphyromonas gingivalis," Frontiers in Bioscience, vol. 5, no. 2, pp. 631-637, 2013.

[36] S. Khan, H. Toyoda, M. Linehan et al., "Poliomyelitis in transgenic mice expressing CD155 under the control of the Tage4 promoter after oral and parenteral poliovirus inoculation," Journal of General Virology, vol. 95, part 8, pp. 1668-1676, 2014. 


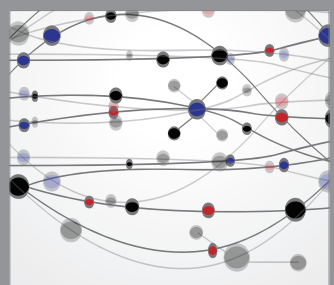

The Scientific World Journal
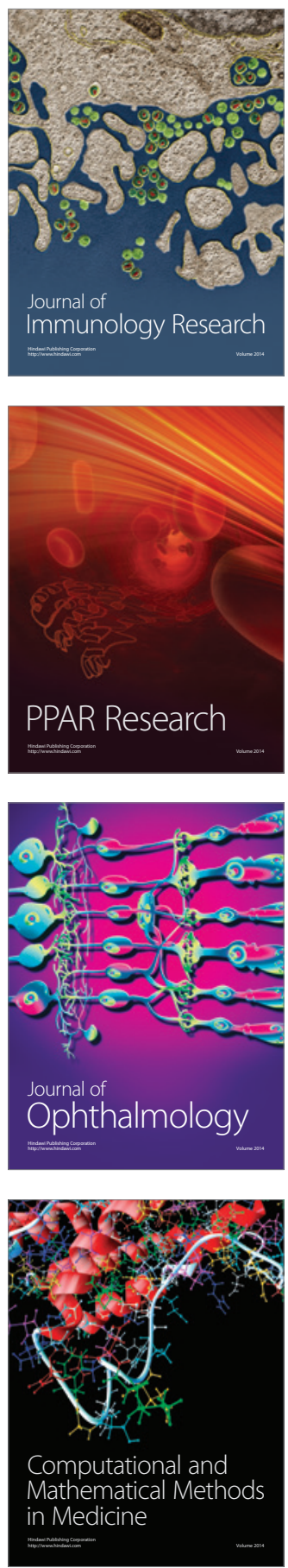

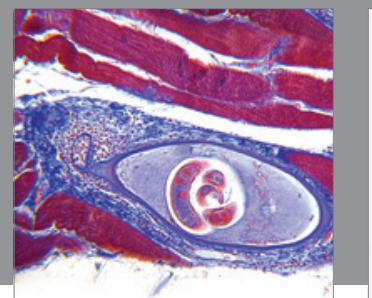

Gastroenterology

Research and Practice
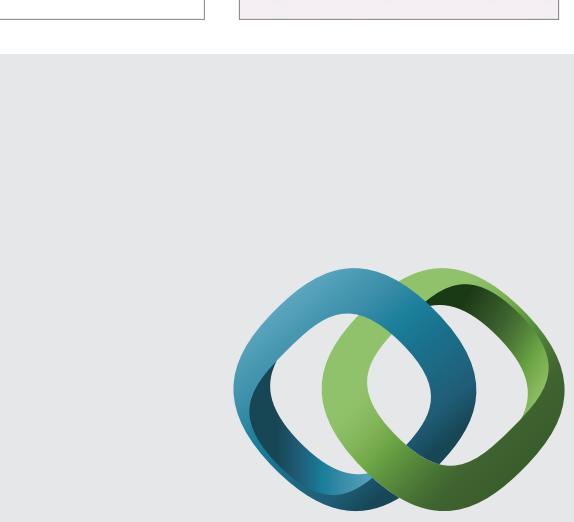

\section{Hindawi}

Submit your manuscripts at

http://www.hindawi.com
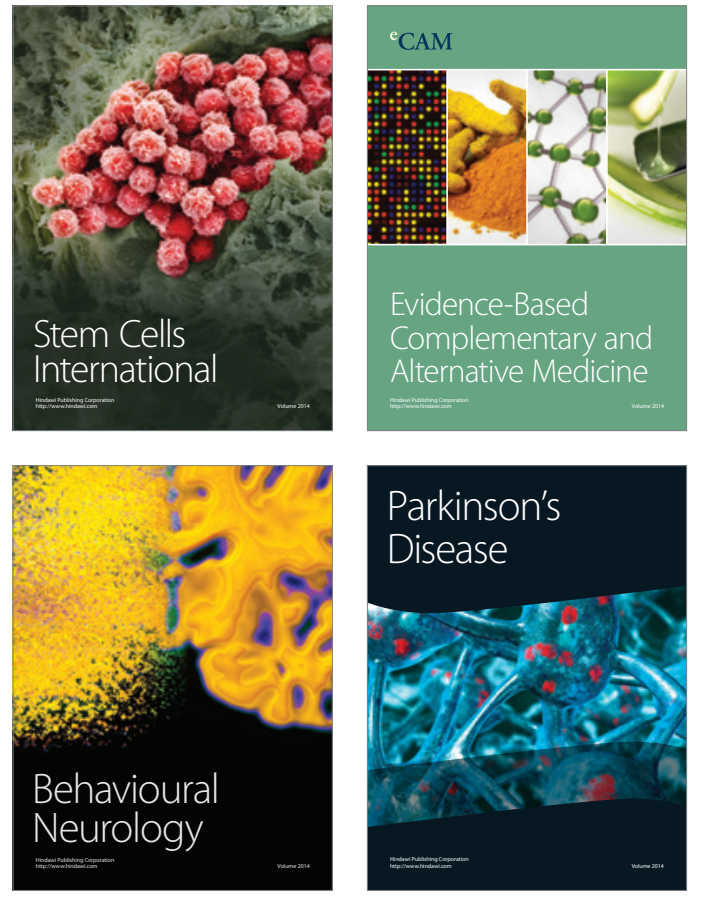
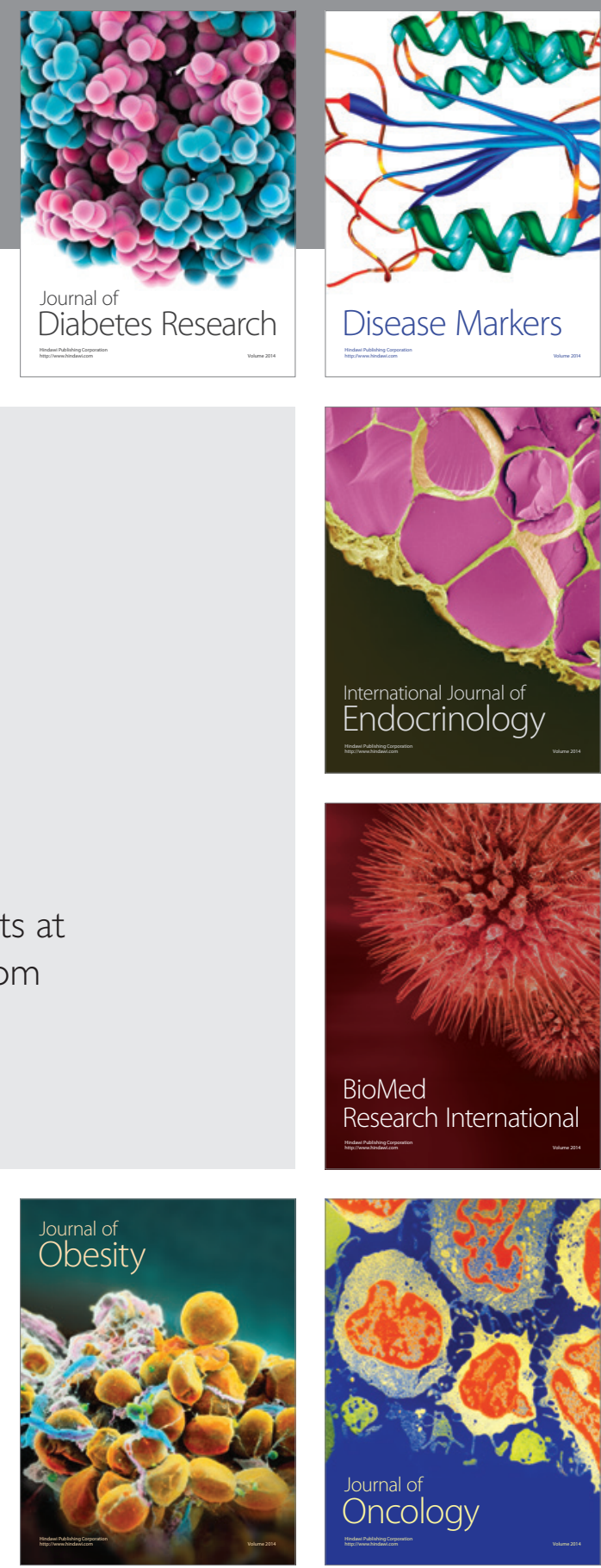

Disease Markers
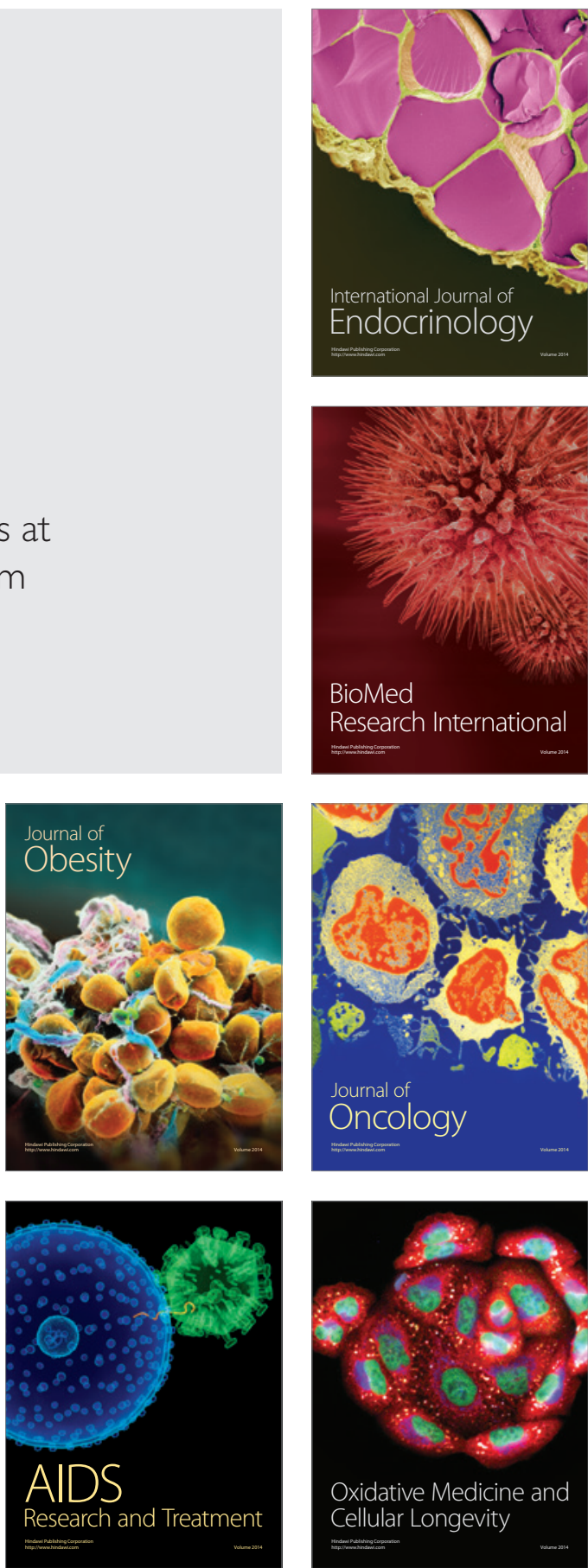